\title{
ANTI-CANCER EFFICIENCY OF NATURAL KILLER CELLS DIFFERENTIATED FROM HUMAN ADIPOSE TISSUE-DERIVED MESENCHYMAL STEM CELLS AND TRANSFECTED WITH MIRNA150
}

\author{
A. Karlitepe ${ }^{1}$, H. Kabadayi', S. Vatansever, ${ }^{2,}$, M. Gurdal', C. Gunduz ${ }^{4}$, G. Ercan ${ }^{1,5, *}$ \\ ${ }^{1}$ Department of Medical Biochemistry, Ege University Medical School, Izmir 35100, Turkey \\ ${ }^{2}$ Department of Histology and Embryology, Celal Bayar University Medical School, Manisa 45030, Turkey \\ ${ }^{3}$ Experimental Health Sciences Research Center, Near East University, Mersin 33010, Turkey \\ ${ }^{4}$ Department of Medical Biology, Ege University School of Medicine, Izmir 35100, Turkey \\ ${ }^{5}$ Department of Stem Cell, Ege University Institute of Health Sciences, Izmir 35100, Turkey
}

\begin{abstract}
Aim: The aim of this study is to investigate the effects of miR150 transfection on NK-like cells differentiated from adipose tissue derived mesenchymal stem cells (AD-MSCs). Methods: NK-like cells were differentiated from AD-MSCs and activated by miR150 transfection. Transfected/non-transfected NK-like cells were characterized by immunohistochemical and RTPCR analyzes. Apoptotic efficiency of the transfected/non-transfected NK-like cells on pancreatic cancer cells PANC1 were determined by TUNEL and RT-PCR. Results: In miR150-transfected cells, the increased expression of NK cell-specific genes such as $G Z M B, K I R 2 D L 2, C D 16, C D 56, N K G 2 D, N K p 46$ and increased immunoreactivity of NK cell-specific surface marker CD314 (NKG2D) were evident. TUNEL assays showed that NK-like cells with/without transfection induced apoptosis in PANC1 cells in the same manner. The decrease in oncogene expression and the increase in the tumor suppressor gene expression in PANC1 cells upon co-culture with NK-like cells differentiated from AD-MSCs were more prominent following miRNA150 transfection. Conclusion: It was shown in vitro that NK-like cells could be obtained by differentiation from AD-MSCs and their efficiency could be increased via miR150 transfection. The results are encouraging for further clinical studies in improvement of immunotherapeutic approaches for cancer therapy.
\end{abstract}

Key Words: pancreatic cancer, adipose tissue derived mesenchymal stem cells, natural killer cells, miRNA150.

Pancreatic cancer $(\mathrm{PaCa})$ is currently the third most frequent cause of cancer-related death in the United States [1]. Pancreatic ductal adenocarcinoma (PDAC), the major histological subtype comprising $90 \%$ of all $\mathrm{PaCa}$, displays local invasion and metastasis during early stages of the disease via developing intrinsic resistance to most therapeutics, contributing to its notoriously poor prognosis with $1-5 \%$ of 5 -year survival rates ( $\sim 6$ months of median survival duration with currently available therapies) [2-4].

Natural killer (NK) cells constitute a significant component of the innate immune system which contributes particularly to the anti-tumor immune response $[5,6]$. NK cells recognize virus-infected and transformed malignant cells and then kill these target cells without prior sensitization [6, 7]. NK cells have various surface receptors (NK cells are CD3-negative, CD16- and/or CD56positive) and cytotoxic granules. NK cells recognize tumor cells and are thought to be good candidates for adaptive immunotherapy against cancer [8]. Recently, they gained attention for their spontaneous cytotoxicity against a broad range of malignancies and for their potential to become a "multi-purpose" anti-cancer

Submitted: August 4, 2017.

*Correspondence: E-mail: gulinnazalper@yahoo.com Tel.: +902323904355

Abbreviations used: AD-MSC - adipose derived mesenchymal stem cell; ESC - embryonic stem cell; HSC - hematopoietic stem cell; MSC - mesenchymal stem cell; NK - natural killer; $\mathrm{PaCa}$ pancreatic cancer; PDAC - pancreatic ductal adenocarcinoma. agent and studies are focused on isolating and expanding NK cells abundantly $[1,6,8]$. NK cells exist in the peripheral blood at a very low concentration and after isolation there is a need to expand them in culture in order to reach a sufficient concentration for clinical applications. However, prolonged culture leads to exhaustion of NK cells and they become ineffective in killing target cells and die within a few days in clinical applications. Therefore, in recent years there have been great interests in attempts to generate NK cells from more abundant cell sources. Until today, NK cells have been differentiated in vitro from different cell sources such as embryonic stem cells (ESCs) or hematopoietic stem cells (HSCs) [6]. NK cells have been also directly isolated from umbilical cord blood or peripheral blood cells [6, 9]. Mesenchymal stem cells (MSCs) are multipotent stem cells with promising potential for cancer research [10-12]. Recently, these cells draw attention because of their importance for tissue microenvironment and their ability of suppressing immune system [11]. They can be obtained from many tissues such as bone marrow and adipose tissue, etc. and are able to be proliferated or differentiated suitably in in vitro conditions $[13,14]$. Usually adipose tissue can be obtained easily and abundantly by a surgical procedure, which is not severely invasive and provides a rich source of MSCs [15, 16]. In addition, adipose derived MSCs (AD-MSCs) have less ethical problems when compared with other sources.

MicroRNAs (miRNAs) are short ( 22 nt) noncoding regulatory RNAs that function as post-transcriptional 
repressors of protein-coding target messenger RNAs and regulate translational repression or degradation $[17,18]$. miR 150 is expressed in various immune cells such as mature NK and innate NK T cells, B and $T$ cells and is up-regulated during cellular maturation [19]. Particularly miR150 is responsible for the development and activation of NK cells and regulates the production of IFNY by NK cells [19].

The aim of this study is to differentiate AD-MSCs to NK-like cells by using miRNA transfection in addition to classical differentiation protocol and then to investigate the effect of these cells on human $\mathrm{PaCa}$ cells in vitro.

\section{MATERIALS AND METHODS}

Cell culture. AD-MSCs purchased from ATCC were cultured in MesenPRO (Thermo Fisher Scientific, MA, USA) medium. Human pancreatic duct endothelial cells (hTERT HPDE) (a kind gift of MD Anderson Cancer Center, TX, USA) were cultured in keratinocyte serum free medium (Thermo Fisher Scientific, MA, USA) supplemented with $10 \%$ fetal bovine serum (FBS), $2 \mathrm{mM}$ L-glutamine, $10 \mathrm{ng} / \mathrm{ml}$ of endothelial growth factor and $1 \%$ penicillin/streptomycin $(\mathrm{P} / \mathrm{S})$ (BI, Cromwell, CT, USA). Human PaCa cell line (Panc1) was obtained from MD Anderson Cancer Center, TX, USA and cultured in DMEM medium supplemented with $10 \%$ FBS, 2 mM L-glutamine and $1 \% \mathrm{P} / \mathrm{S}$. AD-MSC derived NK-like cells were cultured in RPMI- 1640 medium supplemented with $10 \%$ FBS, $12.5 \mathrm{ng} / \mathrm{ml} \mathrm{IL-2,} 5 \mathrm{ng} / \mathrm{ml} \mathrm{IL-15}$ (Sigma, USA) and $1 \% \mathrm{P} / \mathrm{S}$.

Hematopoietic induction. AD-MSC were cultured in Stemline Hematopoietic Stem Cell Expansion Medium (Sigma, USA) supplemented with $1 \%$ bovine serum albumin (BSA), $0.1 \mathrm{mM} 2$-mercaptoethanol, $200 \mu \mathrm{g} / \mathrm{ml}$ of transferrin, $40 \mathrm{mg} / \mathrm{ml}$ of low-density lipoprotein (LDL), $10 \mathrm{mg} / \mathrm{ml}$ of insulin, $10 \mathrm{ng} / \mathrm{ml}$ of thrombopoietin (TPO), $50 \mathrm{ng} / \mathrm{ml}$ of stem cell factor (SCF), $50 \mathrm{ng} / \mathrm{ml} \mathrm{Flt3} \mathrm{ligand,} 20 \mathrm{ng} / \mathrm{ml}$ of granulocytemacrophage-colony stimulating factor (GM-CSF), $20 \mathrm{ng} / \mathrm{ml} \mathrm{IL-3}$, and $20 \mathrm{ng} / \mathrm{ml} \mathrm{IL-6}$ for one week (Sigma, USA). The incubation period lasted for one week while refreshing with the medium which was not supplemented with IL- 6 and TPO on $3^{\text {rd }}$ and $6^{\text {th }}$ days.

NK cell differentiation. Hematopoietically induced-AD-MSCs were cultured in RPMI- 1640 medium supplemented with $50 \mathrm{ng} / \mathrm{ml} \mathrm{SCF}, 50 \mathrm{ng} / \mathrm{ml} \mathrm{Flt-3} \mathrm{li-}$ gand, $12.5 \mathrm{ng} / \mathrm{ml} \mathrm{IL-2,} 20 \mathrm{ng} / \mathrm{ml} \mathrm{IL-7,} 40 \mathrm{ng} / \mathrm{ml} \mathrm{IL-15}$, $2 \mathrm{mM}$ L-glutamine, and $10 \% \mathrm{FBS}$. The incubation period lasted for 4 weeks via refreshing the medium every three days.

MiR 150 transfection. At the end of the $4^{\text {th }}$ week of culturing, AD-MSC-NK cells were transfected with miR150 (Sigma, USA) using a nanoparticle (Xfect) (Takara Bio Inc., USA). In this procedure, AD-MSC-NK cells were incubated with nanoparticlemiRNA complex consisting of miRNA150 and transfection polymer for $48 \mathrm{~h}$ at $37^{\circ} \mathrm{C}$ according to Xfect kit protocol.
Reverse transcription-polymerase chain reaction (RT-PCR). Total RNA was isolated from miR150transfected and non-transfected AD-MSC-NK cells and PANC1 cells before and after co-culturing using RNeasy Mini Kit (Qiagen, Hilden, Germany). Expression of NK cells-specific genes such as GZMB, KIR2DL2, CD16, CD56, NKG2D and NKp46 in NK-like cells and cancer-related genes such as KRas, TP53, CDKN2A, SMAD4, TGFBR2, ARID1A by RT-PCR in PaCa cells was analyzed (Roche, LightCycler 480 Instrument, Basel, Switzerland). The primers used are given in Table.

Table. Primers used for RT-PCR

\begin{tabular}{|c|c|c|}
\hline Gene & Forward primer & Reverse primer \\
\hline GZMB & $\begin{array}{l}\text { 5'-GAAACGCTACTAACTA- } \\
\text { CAGG-3 }\end{array}$ & 5'-CCACTCAGCTAAGAGGT-3 \\
\hline KIR2DL2 & 5'-AGGGTTCTTCTTGCTGC-3' & 5'-AGTGTCCTTAAACTTCCCT-3' \\
\hline CD16 & $\begin{array}{l}\text { 5'-CCCAAGCTTGGGGCC- } \\
\text { GCCACCATGTGGCAGCT- } \\
\text { GCTCCTCCCAACT-3' }\end{array}$ & $\begin{array}{l}\text { 5'-GGGATCCCCAGGTG- } \\
\text { GAAAGAATGATGAGAT-3' }\end{array}$ \\
\hline CD56 & $\begin{array}{l}\text { 5'-TTGTTTTTCCTGGGAACT- } \\
\text { GC-3' }\end{array}$ & $\begin{array}{l}\text { 5'-CCGGATCTGCAGGTAGTT- } \\
\text { GT-3' }\end{array}$ \\
\hline NKG2D & $\begin{array}{l}\text { 5'-TTGACTACTGGACATCTTT- } \\
\text { GCTTTTG-3' }\end{array}$ & $\begin{array}{l}\text { 5'-GAATAATGAGTTTAG- } \\
\text { GAATACAGC-3' }\end{array}$ \\
\hline NKp46 & $\begin{array}{l}\text { 5'-GGCTGTGTCTGAGT- } \\
\text { CAGAG-3' }\end{array}$ & $\begin{array}{l}\text { 5'-GAGTTCATGTCCGGGAT- } \\
\text { GTAG-3' }\end{array}$ \\
\hline KRAS & $\begin{array}{l}\text { 5-AAGGCCTGCTGAAAAT- } \\
\text { GACTG-3' }\end{array}$ & $\begin{array}{l}\text { 5'-CAAAGAATGGTCCTGCAC- } \\
\text { CAG-3' }\end{array}$ \\
\hline TP53 & 5'-AACGGTACTCCGCCACC-3' & 5'-CGTGTCACCGTCGTGGA-3' \\
\hline CDKN2A & $\begin{array}{l}\text { 5'-AGAGGAGGGGCTGGCTG- } \\
\text { GTCA-3' }\end{array}$ & $\begin{array}{l}\text { 5'-CAGCGCCCGCACCTCCTC- } \\
\text { TA-3' }\end{array}$ \\
\hline SMAD4 & $\begin{array}{l}\text { 5'-TTCTAGGTGGCTGGTCG- } \\
\text { GAA-3' }\end{array}$ & $\begin{array}{l}\text { 5'-CAGGTGATACAACTC- } \\
\text { GTTCG-3' }\end{array}$ \\
\hline TGFBR2 & $\begin{array}{l}\text { 5'-TCCGGGAAGGCGCCGTC- } \\
\text { CGCT-3' }\end{array}$ & $\begin{array}{l}\text { 5'-CGACTGTCAAGCGCAGCG- } \\
\text { GAGAG-3' }\end{array}$ \\
\hline$A R I D 1 A$ & 5'-CTTCAACCTCAGT- & 5'- GGTCACCCACCT- \\
\hline Beta Actin & $\begin{array}{l}\text { CAGCTCCCA-3' } \\
\text { 5'-GCACCACACCTTCTA- } \\
\text { CAATG-3' }\end{array}$ & $\begin{array}{l}\text { CATACTCCTTT-3' } \\
\text { 5'-TGCTTGCTGATCCA- } \\
\text { CATCTG-3 }\end{array}$ \\
\hline
\end{tabular}

Immunohistochemical analysis. The cells were fixed with $4 \%$ paraformaldehyde for $30 \mathrm{~min}$ and were washed two times with phosphate buffer saline (PBS). They were then incubated 10 min with $0.1 \%$ Triton $X-100$ solution on ice for permeabilization and $3 \%$ hydrogen peroxide $\left(\mathrm{H}_{2} \mathrm{O}_{2}\right)$ was applied for 5 min after washing with PBS. They were then treated with blocking solution (Invitrogen, CA, USA) for an hour at room temperature and were incubated with primary antibody anti-hNKG2D (RD Systems, MN, USA) overnight at $4{ }^{\circ} \mathrm{C}$. The samples were washed with PBS and incubated with biotinylated rabbit anti-mouse second antibody (Invitrogen, CA, USA) for $30 \mathrm{~min}$. After washing with PBS streptavidin-hydrogen peroxidase (Invitrogen, CA, USA) was added for $30 \mathrm{~min}$. In order to develop the immunohistochemical reaction, diaminobenzidine (DAB) (ScyTek, UT, USA) was applied for $5 \mathrm{~min}$. After washing with PBS, slides were stained with Mayer's hematoxylin and mounted with mounting medium. The intensity of immunolabeling was evaluated by the two investigators in different times with light microscopy (BX40, Olympus, Tokyo, Japan). The immunoreactivities were considered as negative $(-)$, weak $(+)$, moderate $(++)$ and strong $(+++)$.

Co-culture. PANC1 cells were co-cultured with AD-MSC-NK cells using polycarbonate membrane 
(Sigma, MO, USA) in a ratio of 1:2 (NK:PANC1). After incubation for 1 week, the number of dead cancer cells was determined via TUNEL assay.

TUNEL assay. The in situ apoptosis detection kit (ApopTag Plus Peroxidase In Situ Apoptosis Detection Kit, Millipore, MA, USA) was used. The cells were fixed with $4 \%$ paraformaldehyde for $30 \mathrm{~min}$ and were washed two times with PBS. They were then incubated 10 min with $0.1 \%$ Triton $\mathrm{X}-100$ solution on ice for permeabilization and endogenous peroxidase activity was inhibited with $3 \% \mathrm{H}_{2} \mathrm{O}_{2}$. The cells were then incubated with equilibration buffer for 10-15 s and TdT-enzyme in a humidified atmosphere at $37^{\circ} \mathrm{C}$ for $60 \mathrm{~min}$. They were subsequently put into pre-warmed working strength stop/wash buffer at room temperature for $10 \mathrm{~min}$ and incubated with anti-streptavidin-peroxidase for $45 \mathrm{~min}$. Each step was separated by careful washing in PBS. Staining was performed with DAB and counterstaining was performed in Mayer's hematoxylin.

Statistical analysis. Statistical analysis of the data was performed using the Mann - Whitney $U$ test using the SPSS 16 (Statistical Package for the Social Sciences) program. The statistical significance was evaluated, considering $p<0.05$ level as statistically significant.

\section{RESULTS}

Hematopoietic induction and NK-like cell differentiation. AD-MSCs in culture were observed as fusiform cells adherent to the plastic surface (Fig. 1, a). When cultured in hematopoietic induction medium for a week, AD-MSCs detached from plastic surface and became suspended after forming sphere clusters (Fig. 1, b). Hematopoietically induced-AD-MSCs were then cultured in NK differentiation medium for 4 weeks in order to obtain NK-like cells and these cells were called AD-MSC-NK cells (Fig. 1, c).
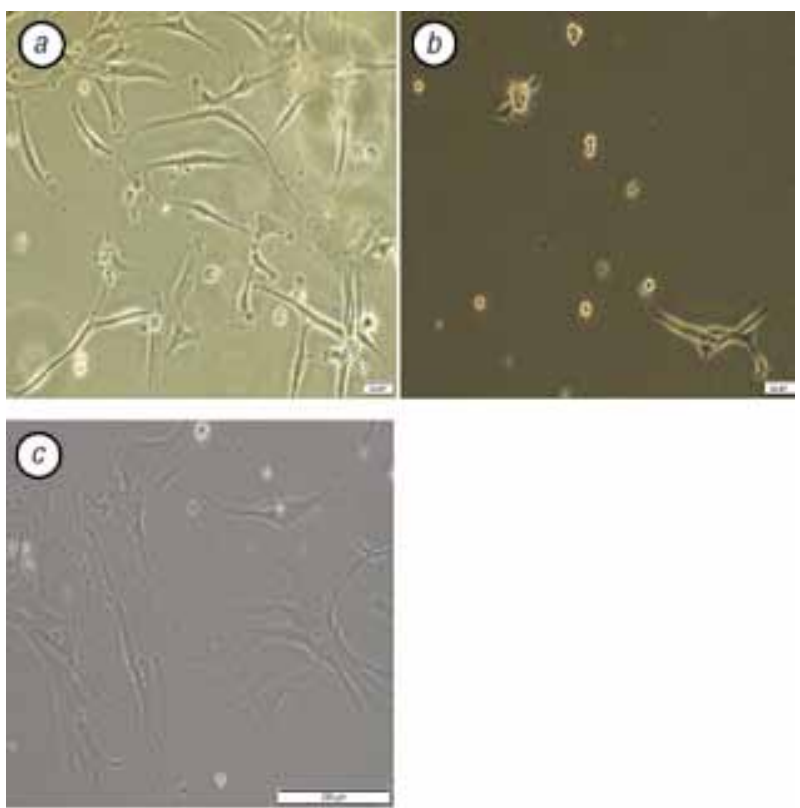

Fig. 1. Characterization of AD-MSC: $a-A D-M S C$ culture; $b-$ hematopoietic induction; $c-A D-M S C-N K$ cell culture

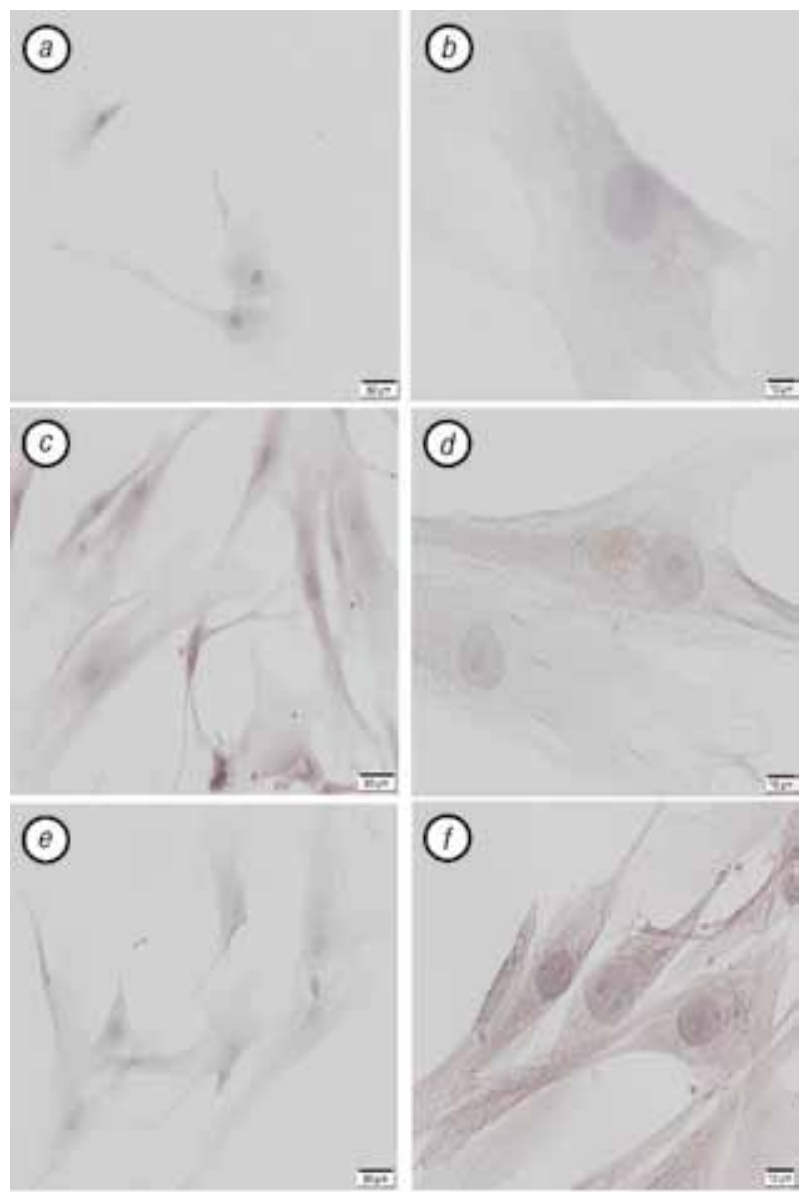

Fig. 2. Characterization of $A D-M S C(a, c, e)$ and $A D-M S C-N K$ $(b, d, f)$ cells. Control staining $(a, b), \operatorname{CD} 90(c, d)$, CD314 (NKG2D) $(e, f)$

Characterization of cultured cells was performed immunohistochemically. Distributions of CD90 and CD314 (NKG2D) were assessed following 4 weeks of culturing of AD-MSCs and AD-MSC-NK cells (Fig. 2). CD90 immunoreactivity was very weakly positive (Fig. 2, e) while NKG2D (CD314) immunoreactivity was strongly positive in AD-MSC-NK cells (Fig. $2, f$ ) at the $30^{\text {th }}$ day of culture. In control slides, negative staining for both CD90 and CD314 was detected (Fig. 2, a, b).

Following miR150 transfection of NK cells differentiated from AD-MSC, NKG2D immunoreactivity was found to be strongly positive for miR150-AD-MSC-NK cells in comparison to non-transfected AD-MSC-NK cells (Fig. 3).

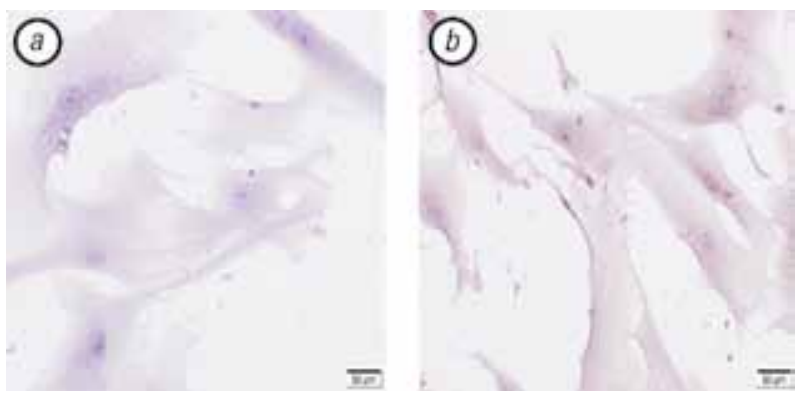

Fig. 3. Immunoreactivity of CD314 (NKG2D) in AD-MSC-NK cells non-transfected (a) and miR150-transfected $(b)$

Expression of NK cell specific genes such as GZMB, KIR2DL2, CD16, CD56, NKG2D and NKp46 significantly increased in AD-MSC-NK cells $(p<0.05)$ when 
compared to undifferentiated AD-MSC as the control group (Fig. 4) and following miR150 transfection, the expression of these genes was found to be even greater in the miR150-transfected AD-MSC-NK cells (miR150-AD-MSC-NK) than the non-transfected AD-MSC-NK cells (AD-MSC-NK) (Fig. 5).

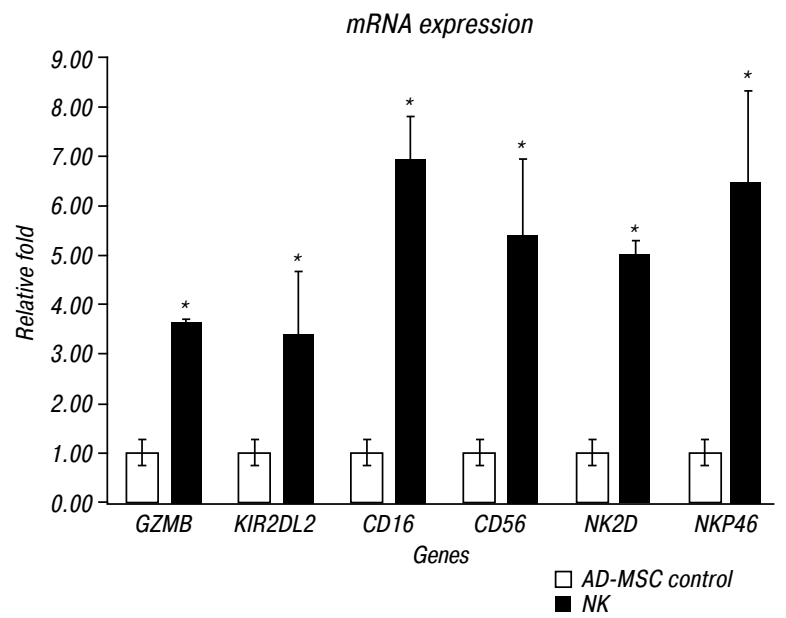

Fig. 4. Variations in gene expressions of $G Z M B$, KIR2DL2, CD16, CD56, NKG2D and NKp46 genes in ADMSC-NK cells in comparison to AD-MSC as the control group (Mean \pm Standard Deviation, Mann - Whitney $U$ test was applied; ${ }^{\star} p<0.05$ ( $\mathrm{n}=3$ for each group)

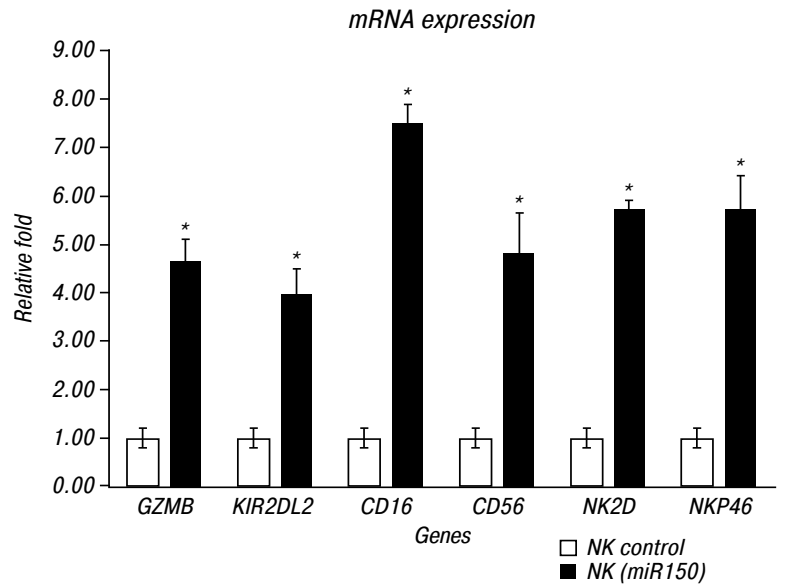

Fig. 5. Variations in gene expressions of $G Z M B, K I R 2 D L 2$, CD16, CD56, NKG2D and NKp46 genes in miR150-transfected AD-MSC-NK cells in comparison to non-transfected AD-MSC-NK cells as the control group (Mean \pm Standard Deviation, Mann - Whitney Utest was applied; ${ }^{*} p<0.05$ ( $\mathrm{n}=3$ for each group)

Induction of apoptosis in PANC1 cells after co-culturing with AD-MSC-NK cells. The TUNEL method based on detection of DNA strand breaks to identify apoptotic cells was used. According to our TUNEL assay results, AD-MSC-NK and miR150-ADMSC-NK were both able to kill PANC1 cancer cells (Fig. 6). TUNEL-positive cells were not detected in the control staining of PANC1 cells, whereas $11 \%$ of TUNEL-positive cells were observed in PANC1 cells after co-culturing with NK cells (Fig. 6, b). After miR150 transfection of NK cells, the percentage of TUNEL-positive cells was $8.8 \%$ in miR150-NKPANC1 co-culture (Fig. 6, d) but only 3.0\% in miR150NK-hTERT co-culture (Fig. $6, f$ ). Only $4 \%$ of TUNEL- positive cells were found in PANC1-AD-MSC coculture (Fig. 6, $h$ ).

After PANC1 cells were co-cultured with AD-MSC-NK cells, the expression of KRAS oncogene significantly decreased $(p<0.05)$, while the expression of tumor suppressor genes such as P53, CDKN2A, TGFBR2, and ARID1A significantly increased $(p<0.05)$ in comparison with that in PANC1 cells before coculturing (Fig. 7). After miR150 transfection, expression of tumor suppressor genes further increased while the oncogene expression was further reduced in PANC1 cells co-cultured with the miR150-transfected AD-MSC-NK cells in comparison to co-culture of PANC1 cells with non-transfected AD-MSC-NK cells (Fig. 8).

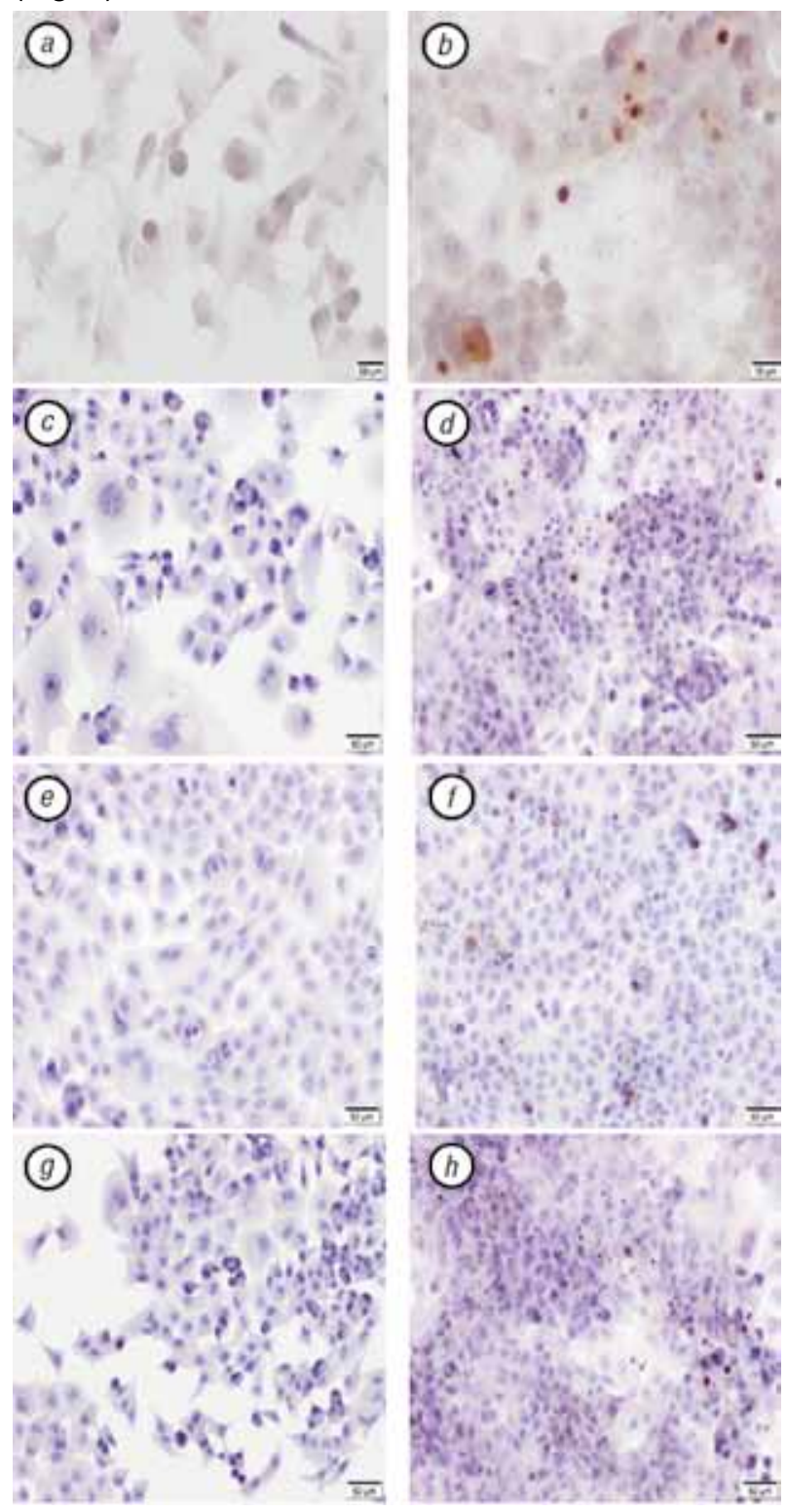

Fig. 6. TUNEL assay: $a-$ control (PANC1); $b-P A N C 1-N K$ co-culture; $c$ - control (PANC1); $d$ - PANC1-miR150-transfected NK coculture; $e$ - control (hTERT-HPDE); $f$ - hTERT-HPDE-miR150-transfected NK co-culture; $g-$ control (PANC1); $h-$ PANC1-AD-MSC co-culture

\section{DISCUSSION}

$\mathrm{PaCa}$, which is frequently seen in the elderly, is an aggressive progressing cancer with 5-year 


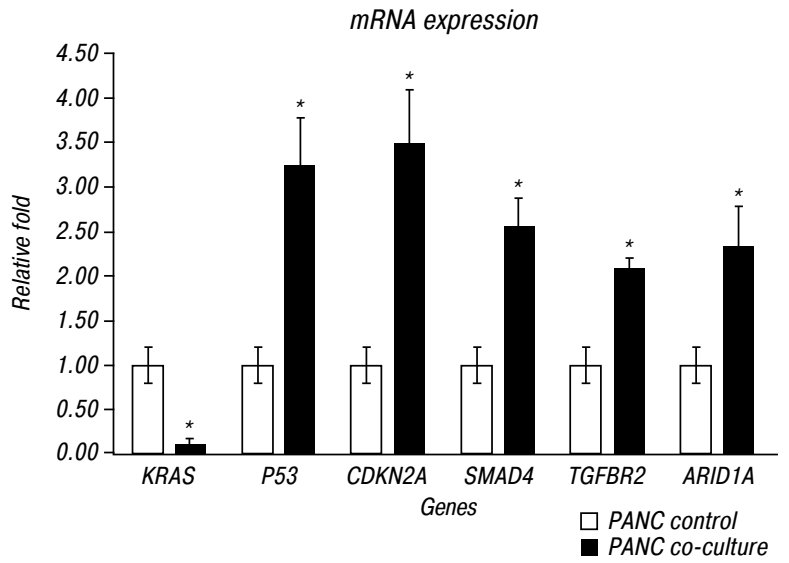

Fig. 7. Variations in gene expressions of KRAS, $P 53, C D K N 2 A$, TGFBR2, and ARID1A genes in PANC1 cells after co-cultured with AD-MSC-NK cells in comparison to the PANC1 cells before co-culturing as the control group (Mean \pm Standard Deviation, Mann - Whitney U test was applied; ${ }^{*} p<0.05$ statistical significance ( $n=3$ for each group)

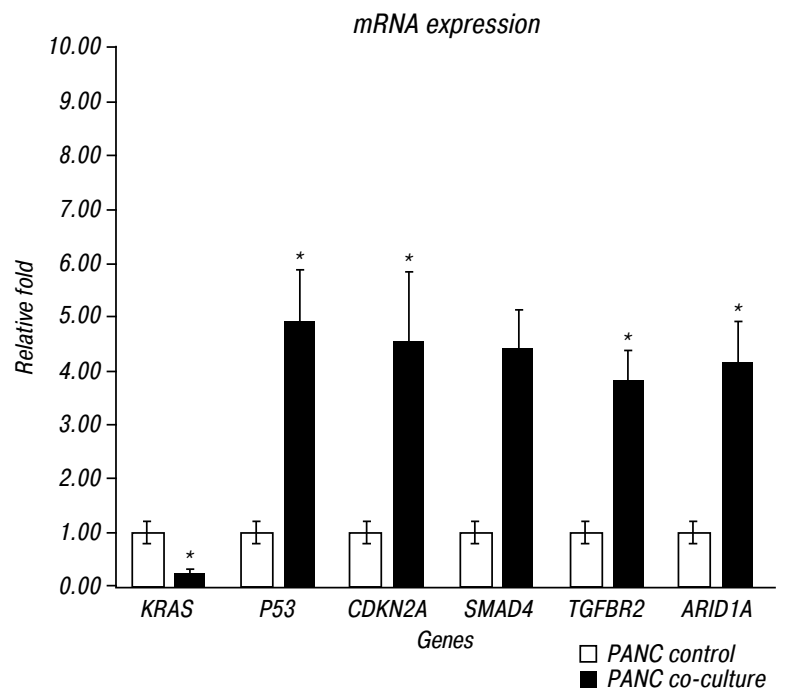

Fig. 8. Variations in gene expressions of KRAS, $P 53, C D K N 2 A$, TGFBR2, and ARID1A genes in PANC1 cells after co-cultured with miR150-transfected AD-MSC-NK cells in comparison to the PANC1 cells after co-culturing with the non-transfected AD-MSC-NK cells as the control group (Mean \pm Standard Deviation, Mann - Whitney $U$ test was applied; ${ }^{\star} p<0.05$ statistical significance ( $n=3$ for each group)

survival rate being less than $5 \%$. In recent years, the incidence of the disease has been increasing steadily and it is in the first place among cancer related deaths $[20,21]$. Among the studies of $\mathrm{PaCa}$, cellbased immunotherapeutic approaches are the most interesting ones. NK cell-based immunotherapeutic approaches in $\mathrm{PaCa}$ have been investigated in phase studies in which NK cells isolated from the donors or the patient were used [22].

In our study, the expression of NK cell-specific genes such as GZMB, KIR2DL2, CD16, CD56, NKG2D, and NKp46 and immunoreactivity of NK cellspecific surface marker CD314 (NKG2D) were evaluated in NK-like cells before and after performing nanoparticle mediated miR150 transfection in ADMSC-NK cells. Both miR150-transfected and nontransfected NK cells were co-cultured with $\mathrm{PaCa}$ cells (PANC1) in order to evaluate their efficiency in killing cancer cells and our results showed that miR150-transfected NK cells and non-transfected NK cells have similar apoptotic activities. However, RT-PCR results indicated that the decrease in gene expressions of oncogenes and the increase in the expression of tumor suppressor genes of $\mathrm{PaCa}$ were more prominent in co-culturing $\mathrm{PaCa}$ cells with miR150-transfected NK cells in comparison to that in co-culturing $\mathrm{PaCa}$ cells with non-transfected NK cells.

The results of the immunohistochemical analysis showed that CD314/NKG2D staining was strongly positive in miR150-NK and AD-MSC-NK cells compared to AD-MSCs. RT-PCR analysis indicated that GZMB, KIR2DL2, CD16, CD56, NKG2D, and NKp46 genes expressed in NK cells were found to be more potent in the miR150-transfected cells.

According to TUNEL results, both miR150-transfected AD-MSC-NK and non-transfected AD-MSCNK-like cells were similarly effective in inducing apoptotic death of PaCa cells. While cells gone to apoptosis can be analyzed by TUNEL assay, the pre-apoptotic cells cannot be analyzed by this assay so we performed Annexin $\mathrm{V}$ in our continuing study and determined $30 \%$ of pre-apoptotic cells in Annexin $V$ assay (data not given).

It should also be noted that there is another pathway leading to cell death named pyroptosis, which is a proinflammatory apoptosis pathway. It will also be valuable to evaluate the pyroptosis pathway in PANC1-NK cells co-culturing via adding caspase- 1 analysis to the procedures already used in our study. IL-18, an inflammatory cytokine associated with NK-like cells, is active in the pathway of pyroptosis, via activating caspase-1, which is not effective in the known apoptosis pathway but is effective in pyroptosis [23].

MSCs are derived from tissues that contain a rich source for the surrounding connective tissue. And thus AD-MSCs are promising immunomodulator stem cells which can be obtained easily and abundantly from several tissues, in particular by liposuction and lipectomy procedures [24, 25], and AD-MSCs do not carry any legal problems as it is an issue for ESC-based studies. Moreover, the cells are easily obtained by getting ethical permission of donors who are subjected to lipectomy procedures voluntarily [26]. Allogeneic NK cells can be easily obtained via differentiation protocol following hematopoietic induction from AD-MSCs and also the efficiency of NK cells can be increased via miRNA transfection. With their high potential as a new immunotherapeutic source, such NK cells will then be available to be used for clinical purposes in the future.

There are many miRNAs that are expressed in human and mouse NK cells [27]. miR150, which regulates the function of NK cells is highly exogenously expressed in resting NK cells in human and 
mice [28]. In our study, NK-like cells differentiated from AD-MSCs were transfected with miR150 using nanoparticles for the first time. RT-PCR analyzes showed that in both non-transfected NK cells and miR150-transfected NK cells oncogene KRAS expression decreased, while expression of tumor suppressor genes (P53, CDKN2A, TGFBR2, SMAD4 and $A R I D 1 A$ increased).

In a recent study, Joshi and colleagues investigated the effect of miRNAs responsible for regulating activation and function of NK cells on melanoma cancer stem cells. In their study, NK cells isolated from the peripheral blood and then transfected with antimiR155 or anti-miR146 both showed antitumorigenic effect on melanoma cells and increased production of antitumorigenic factors released from NK cells such as INFy and granzyme B [29]. In another study, Bezman and colleagues demonstrated that mice with a targeted deletion of miR150 have an impaired, cell lineage - intrinsic defect in their ability to generate mature NK cells while miR150 transgene promotes the development of NK cells, with more mature phenotype and also more responsive to activation indicating that miR150 transfection enhances the function of NK cells [19]. In accordance with these findings, in our study, it was shown that miR150 increased the expression of NK cell-specific genes as it was shown in Fig. 3 and 5.

The efficiency of NK cells derived from HCSs obtained from fresh or frozen cord blood samples was investigated in cancer cell lines using three different culture systems developed by Spanholtz et al. According to their data, the most effective NK cells were obtained from frozen cord blood HSCs and these cells can be used in cancer treatment [30]. A study by Luevano and his team supported these results. It has been reported that NK cells obtained from frozen cord blood HSCs were more effective [9].

Since the activity of NK cells in cancer patients diminishes or disappears with time, cytokines which have potential in activating NK cells such as IL-2, IL-12, IL-15, IL-18, IL-21 are systemically administered to the patients in order to induce endogenous NK cells [31]. However, in these treatment approaches high doses of these cytokines are administered to the patients to strengthen and enumerate NK cells, and it is known that this high dose cytokine treatment has systemic toxic effects [32]. In our study, while obtaining NK-like cells from AD-MSCs which has potential for allogeneic use, we used cytokines at concentrations such as $50 \mathrm{ng} / \mathrm{ml} \mathrm{SCF}, 50 \mathrm{ng} / \mathrm{ml}$ Flt-3 ligand, $12.5 \mathrm{ng} / \mathrm{ml} \mathrm{IL-2,} 20 \mathrm{ng} / \mathrm{ml} \mathrm{IL-7,} 40 \mathrm{ng} / \mathrm{ml} \mathrm{IL-} 15$ for 4 weeks in vitro during the differentiation protocol. In our study, when performing differentiation protocol in vitro we used IL-2 and IL-15 at the nanogram levels which would not lead to systemic toxicity in the future even we will continue to use cytokines at low levels during in vivo studies.

Since $\mathrm{PaCa}$ is one of the most aggressive cancers, our results are promising in improving immunotherapeutic approaches to cure malignancies. The results of this study are encouraging for further clinical studies in improvement of immunotherapeutic approaches for treatment.

\section{ACKNOWLEDGEMENT}

This study was supported by grant No. 214 S650 from the Turkish Scientific and Technological Research Institute (TUBITAK).

\section{REFERENCES}

1. American Cancer Society Pancreatic Cancer overview. https://www.cancer.org/cancer/pancreatic-cancer. 2014.

2. Hajj C, Goodman K. Pancreatic cancer and SBRT: A new potential option? Reports of practical oncology and radiotherapy. Rep Pract Oncol Radiother 2015; 20: 377-84.

3. Ercan G, Karlitepe A, Ozpolat B. Pancreatic cancer stem cells and therapeutic approaches. Anticancer Res 2017; 37: 2761-75.

4. Fitzgerald T, McCubrey J. Pancreatic cancer stem cells: Association with cell surface markers, prognosis, resistance, metastasis and treatment. Adv Biol Reg 2014; 56: $45-50$

5. Shi P, Yin T, Zhou F, et al. Valproic acid sensitizes pancreatic cancer cells to natural killer cell-mediated lysis by upregulating MICA and MICB via the PI3K/Akt signaling pathway. BMC Cancer 2014; 14: 370.

6. Ning H, Lei H, Xu Y, et al. Conversion of adiposederived stem cells into natural killer-like cells with antitumor activities in nude mice. Plos One 2014; 9: e106246.

7. Childs R, Carlsten M. Therapeutic approaches to enhance natural killer cell cytotoxicity against cancer: the force awakens. Nat Rev Drug Discov 2015; 14: 487-98.

8. Peng YP, Zhu Y, Zhang JJ, et al. Comprehensive analysis of the percentage of surface receptors and cytotoxic granules positive natural killer cells in patients with pancreatic cancer, gastric cancer, and colorectal cancer. J Transl Med 2013; 11: 262.

9. Luevano M, Domogala A, Blundell M, et al. Frozen cord blood hematopoietic stem cells differentiate into higher numbers of functional natural killer cells in vitro than mobilized hematopoietic stem cells or freshly isolated cord blood hematopoietic stem cells. Plos One 2014; 9: e87086.

10. Pittenger MF, Mackay AM, Beck SC, et al. Multilineage potential of adult human mesenchymal stem cells. Science 1999; 284: 143-7.

11. Zhao Q, Ren H, Han Z. Mesenchymal stem cells: Immunmodulatory capability and clinical potential in immune diseases. J Cell Immunother 2016; 3: 3-20.

12. Vaananen HK. Mesenchymal stem cells. Ann Med 2005; 37: 469-79.

13. Kern S, Eichler H, Stoeve J, et al. Comparative analysis of mesenchymal stem cells from bone marrow, umbilical cord blood, or adipose tissue. Stem Cells 2006; 24: 1294-301.

14. Rebelatto CK, Aguiar AM, Moretão MP. Dissimilar differentiation of mesenchymal stem cells from bone marrow, umbilical cord blood, and adipose tissue. Exp Biol Med 2008; 233: 901-13.

15. Mareschi K, Biasin E, Piacibello W, et al. Isolation of human mesenchymal stem cells: Bone marrow versus umbilical cord blood. Haematologica 2001; 86: 1099-100.

16. Zuk PA, Zhu M, Ashjian P, et al. Human adipose tissue is a source of multipotent stem cells. Mol Biol Cell 2002; 13: 4279-95.

17. Bezman NA, Cedars E, Steiner DF, et al. Distinct requirements of microRNAs in NK cell activation, survival, and function. J Immunol 2010; 185: 3835-46. 
18. Beaulieu AM, Bezman NA, Lee JE, et al. MicroRNA function in NK cell biology. Immunol Rev 2013; 253: 40-52.

19. Bezman NA, Chakraborty T, Bender T, Lanier L. miR-150 regulates the development of NK and iNKT cells. J Exp Med 2011; 208: 2717-31.

20. Ashour A, Gurbuz N, Alpay S, et al. Elongation factor-2 kinase regulates TG2/b1 integrin/Src/uPAR pathway and epithelial-mesenchymal transition mediating pancreatic cancer cells invasion. J Cell Mol Med 2014; 18: 2235-51.

21. Ashour A, Alpay S, Aziz A, et al. Targeting elongation factor-2 kinase (eEF-2K) induces apoptosis in human pancreatic cancer cells. Apoptosis 2014; 19: 241-58.

22. Kobayashi H, Dubois S, Sato N, et al. Role of transcellular IL-15 presentation in the activation of NK cellmediated killing, which leads to enhanced tumor immunosurveillance. Blood 2005; 105: 721-7.

23. Fink SL, Cookson BT. Apoptosis, pyroptosis, and necrosis: mechanistic description of dead and dying eukaryotic cells. Infect Immun 2005; 73: 1907-16.

24. Wagner W, Wein F, Seckinger A, et al. Comparative characteristics of mesenchymal stem cells from human bone marrow, adipose tissue, and umbilical cord blood. Exp Hematol 2005; 33: 1402-16.
25. Lodi D, Iannitti T, Palmieri B. Stem cells in clinical practice: applications and warnings. J Exp Clin Cancer Res 2011; 30: 9.

26. Silvana G, Lucimar PF, Jerônimo PF, Lydia MF. Characterization of human adipose-derived stem cells. Acta Cirúrgica Brasileira 2012; 27: 471-6.

27. Leong JW, Sullivan RP, Fehniger TA. Natural killer cell regulation by microRNAs health and disease. J Biomed Biotechnol 2012. doi:10.1155/2012/632329.

28. Kim N, Kim M, Yun S, et al. MicroRNA-150 regulates the cytotoxicity of natural killers by targeting perforin-1. J Allergy Clin Immunol 2014; 134: 195-203.

29. Joshi P, Kooshki M, Aldrich W, et al. Expression of natural killer cell regulatory microRNA by uveal melanoma cancer stem cells. Clin Exp Metastasis 2016; 33: 829-38.

30. Spanholtz J, Tordoir M, Eissens D, et al. High logscale expansion of functional human natural killer cells from umbilical cord blood CD34-positive cells for adoptive cancer immunotherapy. Plos One 2010; 5: e9221.

31. Koepsell SA, Miller JS. Natural killer cells: a review of manufacturing and clinical utility. Transfusion 2013; 53: $404-10$.

32. Ni J, Miller M, Stojanovic A, et al. Sustained effector function of IL-12/15/18-preactivated NK cells against established tumors. J Exp Med 2012; 209: 51-65. 